\title{
Improvement in the Excised Citrus Leaf Assay to Investigate Inoculation of 'Candidatus Liberibacter asiaticus' by the Asian Citrus Psyllid Diaphorina citri
}

Laudecir L. Raiol-Junior and Ana D. B. Baia, Universidade Estadual Paulista, Jaboticabal, SP, Brazil; and Fernanda Q. B. F. Luiz, Camila G. Fassini, Viviani V. Marques, and Silvio A. Lopes, Fundecitrus, Araraquara, SP, Brazil

\begin{abstract}
Huanglongbing (HLB) is a difficult-to-control and highly destructive citrus disease that, in Brazil, is associated mainly with the bacterium ' $\mathrm{Can}$ didatus Liberibacter asiaticus' transmitted by the psyllid Diaphorina citri. The aim of this study was to improve our understanding of the ' $\mathrm{Ca}$. L. asiaticus' infection process by exposing excised, fully expanded, immature citrus leaves in 50-ml Falcon tubes to one, four, or eight adults from a ' $\mathrm{Ca}$. L. asiaticus'-exposed colony for 1-, 3-, 7-, or 15-day periods for access to inoculation (IAP). The leaves were incubated at $26^{\circ} \mathrm{C}$ for 1 , $3,7,15$, and 21 days (incubation period [IP]). Infection frequencies and ' $\mathrm{Ca}$. L. asiaticus' titers were assessed by quantitative polymerase chain

reaction (qPCR). ' $\mathrm{Ca}$. L. asiaticus' infection was a function of leaf age, number of insects, IAP, and IP. In general, higher infection rates were observed on younger leaves inoculated with higher numbers of insects and after longer IAP and IP. The immature excised leaf method allowed determination of 3 to 7 days as the range of time required by ' $\mathrm{Ca}$. L. asiaticus' to reach qPCR detectable levels. Even though leaf survival could be prolonged by the maintenance of a branch segment at the base of the leaf petiole, leaf degradation, visible after about 15 days IP, did not allow observation of the entire infection process which, in the intact plant, culminates with the appearance of the blotch mottling symptom on leaf blades.
\end{abstract}

Huanglongbing (HLB) is a highly destructive and difficult-tocontrol citrus disease already reported in the main citrus-producing areas of the world (Bassanezi et al. 2011; Gottwald 2010; Halbert and Manjunath 2004). In Brazil, HLB is associated with pathogens 'Candidatus Liberibacter asiaticus' and ' $\mathrm{Ca}$. L. americanus' (Coletta-Filho et al. 2004; Teixeira et al. 2005b), both transmitted by the psyllid Diaphorina citri (Capoor et al. 1967; Yamamoto et al. 2006). ' $C a$. L. asiaticus' and ' $C a$. L. americanus' occurrences have changed over time in Brazil. In 2004, when the disease was first reported, ' $C a$. L. americanus' was the most prevalent species, found in over 98\% of the HLB-affected trees. Over time, ' $\mathrm{Ca}$. L. asiaticus' replaced ' $\mathrm{Ca}$. L. americanus' and currently has been detected in over $99 \%$ of all the HLB-affected trees that have been analyzed at the Fundecitrus lab (N. A. Wulff, unpublished data). Reasons for this pronounced decrease in ' $\mathrm{Ca}$. L. americanus' occurrence are not known but could be associated with its lower tolerance to higher temperatures and lower ability of the bacterium to reach high populations in citrus as compared with ' $\mathrm{Ca}$. L. asiaticus'. Lower titers may decrease ' $\mathrm{Ca}$. L. americanus' acquisition by the insect vector in a way similar to ' $\mathrm{Ca}$. L. asiaticus' in plants exposed to hot environments (Lopes et al. 2013).

No resistance to HLB has been detected or incorporated into citrus, and diseased trees cannot be cured. There are reports of thermotherapy, antibiotic treatments, and pruning (Hoffman et al. 2013; Lopes et al. 2007; Yang et al. 2016; Zhang et al. 2014). However, most methods are not economical or practically feasible. Therefore, HLB control has relied exclusively on a rigorous and area-wide implementation of the three-pronged system (TPS); namely, removal of all symptomatic trees to reduce sources of inoculum, aggressive control of the insect vector to minimize the chances of Liberibacter transmission, and planting of healthy young trees. The practices involved in TPS have been improved in recent years, including the development of more sensitive polymerase chain reaction (PCR)-based procedures for Liberibacter detection and disease diagnosis, the

Corresponding author: S. A. Lopes; E-mail: silvio.lopes@ fundecitrus.com.br Accepted for publication 13 November 2016.

@ 2017 The American Phytopathological Society development of platforms coupled to tractors for faster and more reliable identification of symptomatic trees in the field, and the development of better ways to monitor and reduce vector populations. Areawide application of TPS has been crucial to suppress disease spread and keep levels in a large area of the Brazilian citrus belt at low disease incidence (Fundecitrus 2016). Despite these advances, success in controlling HLB continues to be a challenge. New practices or improvements of the existing ones would be possible with a deeper understanding of Liberibacter transmission process and spread.

Recently, a method involving excised citrus leaves instead of entire plants to study the process of ' $\mathrm{C}$. . L. asiaticus' acquisition and inoculation by $D$. citri was developed and used by Ammar et al. (2013, 2015). Transmission success was a function of the number of insects carrying ' $\mathrm{Ca}$. L. asiaticus', ' $\mathrm{Ca}$. L. asiaticus' titer in the insects, and time periods for access to ' $\mathrm{Ca}$. L. asiaticus' inoculation and for bacterium incubation in the excised leaves. The main advantages of this method are (i) significant reduction in the time necessary to detect infection in the inoculated citrus (from several months to 1 to 2 weeks) and (ii) reduction in the number and cost of plants used as well as the space needed to conduct these experiments. In this work, a modified version of the excised leaf method for ' $\mathrm{Ca}$. L. asiaticus' inoculation by $D$. citri in citrus was investigated. Using soft, immature leaves instead of mature leaves, higher rates of ' $\mathrm{Ca}$. L. asiaticus' infection were obtained and the time period required by ' $\mathrm{Ca}$. L. asiaticus' to reach quantitative (q)PCR-detectable levels was determined.

\section{Materials and Methods}

Insect sources. A colony of adult $D$. citri from orange jasmine trees grown in the urban area of Santa Fé do Sul municipality, located in northern Sao Paulo State, was used to initiate a mass production of the insects for the experiments. The insects were transferred to the Fundecitrus facilities, confirmed to be free of Liberibacter through qPCR analysis (see below), and reared on new flush growth of symptomatic and ' $\mathrm{Ca}$. L. asiaticus'-positive 5-year-old potted 'Valencia' sweet orange on Rangpur lime rootstock. To increase the chances that most insects to be used in the inoculation experiments were ' $C a$. L. asiaticus' positive, all individuals were reared for at least two generations on the infected plants at $25^{\circ} \mathrm{C}( \pm 3)$ with a 12-h photoperiod, inside a programmable growth chamber (Conviron), where all the experiments were also carried out. Before each experiment, 
combined samples of five individuals were further tested by qPCR to determine their suitability for ' $\mathrm{C} a$. L. asiaticus' inoculation in terms of ' $\mathrm{Ca}$. L. asiaticus' titer in the insect population.

Leaf sources. All leaves used in the experiments were collected from a group of 12 healthy trees of 3-year-old potted Valencia sweet orange grafted on Rangpur lime maintained inside an insect-proof greenhouse, with a daily average of $29.8^{\circ} \mathrm{C}( \pm 3)$, and $76 \%( \pm 10)$ relative humidity. The trees were irrigated and fertilized appropriately and pruned as needed to produce leaves at the appropriate growth stages.

Excised-leaf procedures. A modified version of the excised-leaf method of Ammar et al. (2013) was used in most experiments. Fully expanded soft leaves, measuring 5.5 to $6.5 \mathrm{~cm}$ in length by 2.5 to $3.5 \mathrm{~cm}$ wide, were removed with a razor blade from 3- to 4-weekold branches. The cut was made in a way to keep a 1-cm-long segment of the branch attached to the leaf petiole (Fig. 1). In preliminary tests, maintaining the 1-cm-long branch segment increased leaf longevity. Branch segment plus leaf petiole were then immediately introduced into a hole made in the center of an 8-cm-diameter disc of Whatman filter paper, and introduced into a 50-ml propylene Falcon tube containing $15 \mathrm{ml}$ of sterile water. Then, 15-day-old adult psyllids were confined on each leaf for varying periods of time, depending on the experimental purpose. Screened mesh nylon tissue replaced the tube cap to allow air flow and favor insect survival. All experiments were carried out inside the programmable growth chamber under the conditions already described. The visual aspect of the leaves was evaluated daily during the time that experiments were conducted. At the end of each incubation period (IP), the leaves were individually processed for DNA extraction and qPCR analysis (see below).

Inoculation experiments. Three experiments were carried out. The first experiment was conducted to determine whether the age of the leaf would affect ' $\mathrm{Ca}$. L. asiaticus' transmission. Batches of five adult ' $\mathrm{Ca}$. L. asiaticus'-exposed insects were confined in 50-ml Falcon conical tubes, each containing either a fully expanded light-green immature leaf or a fully expanded dark-green mature leaf. Both types of leaves were removed from the same mother plants and prepared the same way as indicated. The insects remained caged for 1,3 , or 7 days for access to ' $\mathrm{Ca}$. L. asiaticus' inoculation (inoculation access period [IAP]) and remained extra days, when needed, to complete a total of 7 days for ' $\mathrm{Ca}$. L. asiaticus' incubation (IP). In this and all remaining experiments, the number of days of IP was calculated by including the number of days of IAP. In the first experiment, the effectiveness to inoculate immature and mature leaves was
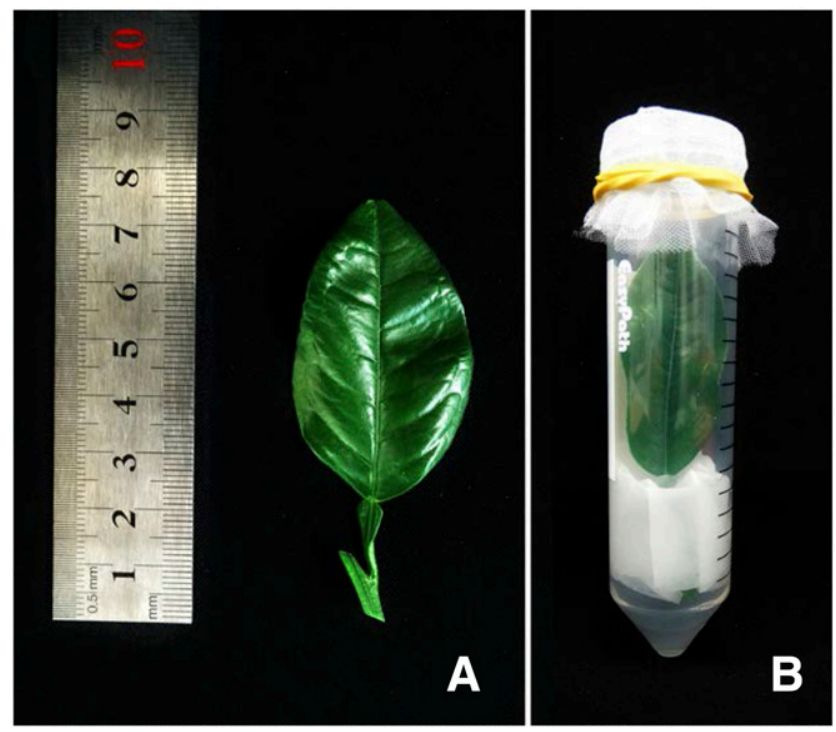

Fig. 1. Aspect of $\mathbf{A}$, an immature fully expanded citrus leaf and $\mathbf{B}$, the same leaf enclosed into a Falcon tube containing $15 \mathrm{ml}$ of sterile water, used in all 'Candidatus Liberibacter asiaticus' inoculation experiments (modified from Ammar et al. 2013) assessed using the same lots of insects. The psyllids used for IAP of immature leaves were collected and used for IAP of mature leaves and vice versa. qPCR was employed also to determine the ' $C a$. L. asiaticus' titer in the lots of insects used for inoculation.

The second experiment was conducted to determine (i) the minimum number of insects and the minimum IAP necessary for successful ' $\mathrm{Ca}$. L. asiaticus' inoculation and (ii) whether ' $\mathrm{Ca}$. L. asiaticus' would multiply in the excised leaves. One, four, or eight adult ' $\mathrm{Ca}$. $\mathrm{L}$. asiaticus'-exposed insects were confined on immature leaves inside Falcon tubes. The insects were maintained for $1,7,15$, or 21 days of IAP, and the leaves were maintained for 7, 15, or 21 days of IP. This experiment was repeated twice but only with four psyllids per leaf, with IAP of 1 and 3 days and IP of 7 and 15 days. As in the first experiment, ' $\mathrm{Ca}$. L. asiaticus' titer was assessed in the lots of insects used for inoculation.

The third experiment was conducted to determine how effective the excised immature leaf method was compared with intact leaves (maintained attached to the plant) for successful ' $\mathrm{Ca}$. L. asiaticus' inoculation. Two fully expanded soft leaves that developed on opposite sides at the top of a branch on 3-month-old potted Valencia/'Cravo' Rangpur lime plants were selected. The upper leaf was removed from the branch and transferred into a Falcon tube as indicated. The next lower leaf located on the opposite side was maintained intact, attached to the branch (nonexcised leaf). Lots of five ' $\mathrm{Ca}$. L. asiaticus'-exposed $D$. citri adults were then confined on the excised leaf inside the tubes and on the nonexcised leaf; in this instance, inside a 15-by-10-cm screened bag, made of the same voile tissue used to replace the cap of the Falcon tube. Excised and nonexcised leaves were exposed to the same lots of insects as indicated in the first experiment. The insects were maintained for 3 days of IAP and 7 days of IP. This experiment was repeated once.

DNA extraction and qPCR analysis. DNA was extracted from individual insects, lots of variable numbers of insects, or $0.5 \mathrm{~g}$ of chopped plant tissues (leaf midribs), as described by Teixeira et al. (2005a). qPCR was processed in total volumes of $12 \mu \mathrm{l}$ containing the master mix, $1 \mu$ l of plant tissue DNA $(100 \mathrm{ng} / \mu \mathrm{l})$ or $5 \mu \mathrm{l}$ of nonquantified insect DNA, and $150 \mathrm{nM}$ each of primers HLBaspr, in a StepOne Plus thermocycler (Applied Biosystems), following the cycling protocol of (Li et al. 2006). Quantification cycle threshold values were automatically adjusted using the StepOne software and the quantity of amplified DNA converted in log of DNA copies per gram of leaf tissue or per individual or groups of insect, as indicated (Lopes et al. 2013).

Statistical analysis. All experiments were carried out in completely randomized designs. The proportion of ' $\mathrm{Ca}$. L. asiaticus'-positive leaves in relation to ' $\mathrm{Ca}$. L. asiaticus'-negative leaves was analyzed by the $\chi^{2}$ test using Statistica software (StatSoft, Tulsa, OK).

\section{Results}

Influence of leaf age on the efficiency of ' $\mathrm{Ca}$. L. asiaticus' inoculation. We initially compared the suitability of soft immature leaves with that of mature leaves for ' $\mathrm{Ca}$. L. asiaticus' inoculation by caging five adults ' $\mathrm{Ca}$. L. asiaticus'-exposed psyllids on leaves for 1, 3, and 7 days of IAP. Higher infection frequencies were observed on immature $(29.2 \%)$ than mature $(8.3 \%)$ leaves (Table 1$)$.

Table 1. Suitability of excised citrus leaves at two developmental stages for successful inoculation of 'Candidatus Liberibacter asiaticus' by groups of five Diaphorina citri adults after three inoculation access periods (IAP)

\begin{tabular}{|c|c|c|c|c|}
\hline \multirow[b]{3}{*}{ IAP (days) } & \multicolumn{4}{|c|}{ Fully expanded leaf } \\
\hline & \multicolumn{2}{|c|}{ Light-green immature } & \multicolumn{2}{|c|}{ Dark-green mature } \\
\hline & Frequency $^{\mathbf{a}}$ & Average $\pm \mathbf{S E}^{\mathbf{b}}$ & Frequency $^{a}$ & Average $\pm \mathbf{S E}^{b}$ \\
\hline 1 & $1 / 16(6.3)$ & 2.29 & $1 / 16(6.3)$ & 2.68 \\
\hline 3 & $5 / 16(31.0)$ & $2.87 \pm 0.41$ & $2 / 16(12.5)$ & $2.77 \pm 0.30$ \\
\hline 7 & $8 / 16(50.0)$ & $3.32 \pm 0.65$ & $1 / 16(6.3)$ & 2.32 \\
\hline Averages & $14 / 48(29.2)$ & $2.83 \pm 0.30$ & $4 / 48(8.3)$ & $2.59 \pm 0.14$ \\
\hline
\end{tabular}

${ }^{a}$ Frequency and percentage of quantitative polymerase chain reaction-positive samples with quantification cycle values $\leq 34.0$.

b Average \pm standard error (SE) of log ' $\mathrm{Ca}$. L. asiaticus' cells per gram of tissue. 
Caging the insects on immature and then on mature leaves and vice versa did not affect the results. On immature leaves, the infection frequency increased gradually with the increase in IAP. On mature leaves, however, an increase was detected only from 1 to 3 days of IAP. Contrary to the infection frequency, longer IAP did not result in higher ' $\mathrm{Ca}$. L. asiaticus' titer in the leaves. Prior to the start of the experiment, all lots of insects carried ' $\mathrm{Ca}$. L. asiaticus', with titers of 2.55 to 6.15 ( $n=16$, average $4.33 \pm 0.17)$ per lot of five insects. At the end of each IAP, the percentage of ' $\mathrm{Ca}$. L. asiaticus'-positive samples ranged from 6.3 to 50 for the immature and 6.3 to 12.5 for the mature leaves.

Influence of insect number and IAP. Because immature leaves resulted in higher frequencies of ' $\mathrm{Ca}$. L. asiaticus' infection, they were used in further experiments. Subsequently, we tried to determine whether the success of ' $\mathrm{Ca}$. L. asiaticus' inoculation would increase with an increase in the number of insects used for inoculation and in the period of time the insects were allowed to feed on the leaves (IAP). Also, we tried to determine whether longer IP would result in higher ' $\mathrm{Ca}$. L. asiaticus' titer in the inoculated leaves.

After 15 days of IP, some excised leaves started losing the original light-greenish color and becoming dark yellow, when they were discarded from the experiment. As a result, the number of leaves and insects that remained until the end of the experiment and were assessed by qPCR decreased from the original 10 to 3 to 5 at the 21 st day of IP.

In general, the use of higher numbers of insects resulted in significantly higher frequencies of qPCR-positive leaves $\left(\chi^{2}=11.24, \mathrm{df}=2\right.$, $P<0.0036)$. This occurred for all three IAP and most IP tested (Table 2). On average, one and eight insects transmitted ' $\mathrm{Ca}$. L. asiaticus' to 21.8 and $57.8 \%$ of the leaves after 1 day of IAP, 46.0 and $75.7 \%$ after 7 days of IAP, and 32.0 and $66.5 \%$ after 15 days of IAP, respectively. No significant influence of insect number on ' $\mathrm{Ca}$. L. asiaticus' titer in the inoculated leaves was detected. Similarly, no influence of the IP on the frequency of ' $\mathrm{Ca}$. L. asiaticus' infection $\left(\chi^{2}=1.64, \mathrm{df}=1, P<0.16\right)$ was detected.

This experiment was repeated twice, with higher numbers of replications (15 and 30 per treatment in experiments 1 and 2, respectively), except caging four insects per leaf for 1 and 3 days of IAP. The leaves were assessed for qPCR after 7 and 15 days of IP (Table 3). Most leaves retained their original light yellow color to the end of each evaluation period. The overall frequency of successful inoculation was higher in experiment 1 . Similar to the previous experiment (Table 2), no statistical differences were detected between infection frequencies and ' $C a$. L. asiaticus' titers at 7 and 15 days of IP.

Excised versus nonexcised leaves. The efficiency of excised leaves was compared with that of nonexcised leaves in two experiments; namely, leaves of the same age and similar appearance but maintained intact and attached to the plant for successful
' $C a$. L. asiaticus' inoculation (Table 4). In experiment 1, we used one insect; 1 day of IAP; and 1, 3, or 7 days of IP. In experiment 2, we used four insects, 3 days of IAP, and 7 or 15 days of IP.

All excised leaves in experiment 1 retained the normal aspect and were assessed by qPCR but, in experiment 2,5 of 20 had to be removed after 15 days of IAP due to changes in the leaf blade, similar to those described earlier. On nonexcised leaves, the frequency of successful inoculation remained the same after 3 and 7 days of IP in experiment 1 . In experiment 2 , ' $\mathrm{Ca}$. L. asiaticus' was detected after 7 but not 15 days of IP. Overall, no statistical differences were detected in frequencies of infection and ' $\mathrm{Ca}$. L. asiaticus' titers between excised and nonexcised leaves and IP in both experiments.

\section{Discussion}

Improvements in HLB management practices require additional understanding of the complex interaction involving the citrus plant, the HLB pathogen, and the insect vector. Recently, an excised leaf method was developed to facilitate such studies (Ammar et al. 2013). A significant reduction in time, cost, and space required to conduct these experiments are the main advantages of this method. We developed a modified version of the Ammar method with immature and mature expanded leaves. Infection frequencies were higher on immature leaves, probably because they are the preferred feeding sites of D. citri (Tsai and Liu 2000).

In attempts to establish the most appropriate conditions for ' $\mathrm{Ca}$. $\mathrm{L}$. asiaticus' transmission, a series of experiments was carried out using soft immature leaves. The frequency of successful inoculation

Table 3. Suitability of excised fully expanded, light-green immature citrus leaves for successful inoculation of 'Candidatus Liberibacter asiaticus' by four Diaphorina citri adults after two periods for access to inoculation (IAP) and bacterial incubation (IP)

\begin{tabular}{llllll}
\hline & \multicolumn{2}{c}{ One-day IAP } & & \multicolumn{2}{c}{ Three-day IAP } \\
\cline { 2 - 3 } \cline { 5 - 6 } IP (days) & $\begin{array}{c}\text { Frequency } \\
(\boldsymbol{\%})^{\mathbf{a}}\end{array}$ & $\begin{array}{c}\text { Average } \pm \\
\mathbf{S E}^{\mathbf{b}}\end{array}$ & & $\begin{array}{c}\text { Frequency } \\
(\boldsymbol{\%})^{\mathbf{a}}\end{array}$ & $\begin{array}{c}\text { Average } \pm \\
\mathbf{S E}^{\mathbf{b}}\end{array}$ \\
\hline Experiment 1 & & & & \\
$\quad 7$ & $3 / 15(20)$ & $3.54 \pm 0.46$ & & $2 / 15(13)$ & $2.93 \pm 0.31$ \\
$\quad 15$ & $4 / 15(27)$ & $3.39 \pm 0.47$ & & $4 / 12(33)$ & $3.12 \pm 0.10$ \\
$\quad$ Average & $7 / 30(23.4)$ & 3.47 & & $6 / 27(22.2)$ & 3.03 \\
Experiment 2 & & & & \\
$\quad 7$ & $3 / 30(10)$ & $3.51 \pm 0.61$ & & $1 / 30(3)$ & 4.09 \\
$\quad 15$ & $6 / 30(20)$ & $2.68 \pm 0.14$ & & $2 / 28(8)$ & $2.64 \pm 0.31$ \\
$\quad$ Average & $9 / 60(15.0)$ & 3.10 & & $3 / 58(5.2)$ & 3.37 \\
\hline
\end{tabular}

${ }^{a}$ Frequency and percentage of quantitative polymerase chain reaction-positive samples with quantification cycle values $\leq 34.0$.

${ }^{\mathrm{b}}$ Average \pm standard error (SE) of $\log$ ' $\mathrm{Ca}$. L. asiaticus' cells per gram of tissue.

Table 2. Suitability of excised fully expanded, light-green immature citrus leaves for successful inoculation of 'Candidatus Liberibacter asiaticus' by one, four, or eight Diaphorina citri adults after three periods for access to inoculation (IAP) and four periods for bacterial incubation (IP)

\begin{tabular}{|c|c|c|c|c|c|c|c|c|c|}
\hline \multirow[b]{3}{*}{ IP (days) } & \multicolumn{9}{|c|}{ IAP and number of adult psyllids } \\
\hline & \multicolumn{3}{|c|}{ 1-day IAP } & \multicolumn{3}{|c|}{ 7-day IAP } & \multicolumn{3}{|c|}{ 15-day IAP } \\
\hline & 1 & 4 & 8 & 1 & 4 & 8 & 1 & 4 & 8 \\
\hline \multicolumn{10}{|l|}{ Freq $(\%)^{\mathrm{a}}$} \\
\hline 1 & $0 / 10(0)$ & $0 / 10(0)$ & $1 / 10(10)$ & $\ldots$ & $\ldots$ & $\ldots$ & $\ldots$ & $\ldots$ & $\ldots$ \\
\hline 7 & $3 / 10(30)$ & $4 / 10(40)$ & $6 / 10(60)$ & $8 / 10(80)$ & 9/10 (90) & $10 / 10(100)$ & $\cdots$ & $\ldots$ & $\ldots$ \\
\hline 15 & 1/7 (14) & $2 / 8(25)$ & $5 / 7(71)$ & $3 / 8(38)$ & $6 / 10(60)$ & $6 / 9(67)$ & 4/9 (44) & $4 / 6(67)$ & $5 / 6(83)$ \\
\hline 21 & $2 / 5(40)$ & $3 / 5(60)$ & $4 / 5(80)$ & $1 / 5(20)$ & $3 / 5(60)$ & $3 / 5(60)$ & $1 / 5(20)$ & $0 / 3(0)$ & $2 / 4(50)$ \\
\hline Averages & $21.8 \pm 9.1$ & $32.3 \pm 12.9$ & $57.0 \pm 15.6$ & $46.0 \pm 17.8$ & $70.0 \pm 10.0$ & $75.7 \pm 12.3$ & $32.0 \pm 12.0$ & 33.5 & $66.5 \pm 16.5$ \\
\hline \multicolumn{10}{|l|}{ Titer $^{\mathrm{b}}$} \\
\hline 1 & 0 & 0 & 2.3 & & & $\ldots$ & $\ldots$ & $\ldots$ & $\ldots$ \\
\hline 7 & 2.3 & 3.1 & 2.8 & 2.5 & 2.7 & 3.0 & $\ldots$ & $\ldots$ & $\ldots$ \\
\hline 15 & 3.1 & 3.9 & 2.8 & 2.8 & 2.6 & 3.1 & 3.2 & 2.5 & 3.6 \\
\hline 21 & 2.4 & 2.7 & 3.2 & 2.2 & 2.6 & 3.4 & 3.9 & 0 & 2.5 \\
\hline Averages & $2.0 \pm 0.7$ & $2.4 \pm 0.8$ & $2.8 \pm 0.2$ & $2.5 \pm 0.2$ & $2.6 \pm 0.03$ & $3.2 \pm 0.1$ & $3.6 \pm 0.4$ & $1.3 \pm 1.3$ & $3.1 \pm 0.6$ \\
\hline
\end{tabular}

${ }^{a}$ Frequency and percentage of ' $C a$. L. asiaticus'-positive leaves; samples with quantification cycle values $\leq 34.0$.

b 'Ca. L. asiaticus' titer ( $\log _{10}$ bacterium cells per gram of leaf midrib). 
increased with an increase in the number of insects and, in general, with an increase of leaf exposure time to ' $\mathrm{Ca}$. L. asiaticus'-exposed insects, reflecting an overall inoculum dose effect, also reported by Ammar et al. (2013). Higher numbers of ' $C a$. L. asiaticus'-positive individuals feeding for longer periods would increase the chances of injecting higher numbers of bacterium cells into the phloem.

In our studies, an increase in the frequency of successful inoculation was also observed with an increase in the IP from 1 to 21,7 to 15 , and 1 to 7 days (Tables 2, 3, and 4, respectively). This effect was observed when variable numbers of insects were used for inoculation but with IAP less than 3 days. Longer IAP ( 7 and 15 days), in fact, resulted in lower frequencies of successful inoculation, probably as a result of internal leaf damage induced by the insect-feeding process providing, in turn, a less favorable environment for ' $\mathrm{Ca}$. L. asiaticus' multiplication.

The increase in the frequency of successful inoculation with longer IP may be the result of ' $\mathrm{Ca}$. L. asiaticus' multiplication in the leaf tissues during an initial infection process, increasing the titer from values below to above the qPCR detection limit. In our hands, using Li's protocol ( $\mathrm{Li}$ et al. 2006), the qPCR detection limit was estimated as $2.2 \mathrm{log}$ cells per gram of tissue, equivalent to a 34.0 quantification cycle value. Because the increase in the frequency of infection was not followed by a consistent increase in ' $\mathrm{Ca}$. L. asiaticus' titer, which apparently reached a plateau in the range of three to four log cells per gram of tissue after 3 to 7 days of incubation (Table 4), these values seem to be the upper titer limits that ' $\mathrm{Ca}$. L. asiaticus' would reach in detached leaves. These values are three to four log values lower than those detected on mature citrus leaves of graft-inoculated potted plants expressing the typical HLB blotchy mottle symptom, or even on asymptomatic leaves, 3 to 4 months after inoculation (Lopes et al. 2009).

In attempts to further determine the potential of excised leaves to replace entire plants in ' $\mathrm{Ca}$. L. asiaticus' inoculation experiments, excised immature leaves were compared with nonexcised immature leaves of the same developmental and physiological stage that remained attached to the original plant in two experiments varying the number of insects, IAP, and IP. The frequency of successful ' $C a$. L. asiaticus' inoculation was lower for whole plants (nonexcised leaf treatment) in both experiments but did not differ statistically from that of excised leaves. The relatively short longevity of the detached leaves did not allow assessment of infection progress. Interestingly, the few plants in which ' $\mathrm{Ca}$. L. asiaticus' was detected in the attached inoculated leaves after 3 and 7 days of IP did not develop HLB symptoms, and the bacterium was not detected by qPCR carried out at 8 months after inoculation. The reason for the lack of progression of ' $C a$. L. asiaticus' infection to the whole plant is not known but could be the result of an effective "pruning

Table 4. Suitability of excised and nonexcised fully expanded, light-green immature citrus leaves for successful inoculation of 'Candidatus Liberibacter asiaticus' by Diaphorina citri adults after variable inoculation access (IAP) and incubation (IP) periods

\begin{tabular}{|c|c|c|c|c|}
\hline \multirow[b]{2}{*}{ IP (days) } & \multicolumn{2}{|c|}{ Excised leaves } & \multicolumn{2}{|c|}{ Nonexcised leaves } \\
\hline & $\begin{array}{c}\text { Frequency } \\
(\%)^{\mathrm{a}}\end{array}$ & $\begin{array}{c}\text { Average } \\
\pm \mathrm{SE}^{\mathrm{b}}\end{array}$ & $\begin{array}{c}\text { Frequency } \\
(\%)^{\mathrm{a}}\end{array}$ & $\begin{array}{c}\text { Average } \\
\pm \mathbf{S E}^{\mathbf{b}}\end{array}$ \\
\hline \multicolumn{5}{|c|}{$\overline{\text { Experiment } 1^{\mathrm{c}}}$} \\
\hline 1 & $0 / 20(0)$ & 0 & 0/20 (0) & 0 \\
\hline 3 & $2 / 20(10)$ & $3.14 \pm 0.31$ & $2 / 20(10)$ & $3.40 \pm 0.04$ \\
\hline 7 & $4 / 20(20)$ & $3.61 \pm 0.12$ & $2 / 20(10)$ & $3.20 \pm 0.12$ \\
\hline Average & $6 / 60(10.0)$ & 2.25 & $4 / 60(6.7)$ & 2.2 \\
\hline \multicolumn{5}{|c|}{ Experiment $2^{\mathrm{d}}$} \\
\hline 7 & $2 / 20(10)$ & $3.00 \pm 0.5$ & $1 / 20(5)$ & 3.90 \\
\hline 15 & $2 / 15$ & $2.30 \pm 0.3$ & $0 / 20(0)$ & 0 \\
\hline Average & $4 / 35(11.4)$ & 2.70 & $1 / 40(2.5)$ & 1.95 \\
\hline
\end{tabular}

a Frequency and percentage of quantitative polymerase chain reaction-positive samples with quantification cycle values $\leq 34.0$.

b Average \pm standard error (SE) of log ' $\mathrm{Ca}$. L. asiaticus' cells per gram of tissue.

${ }^{c}$ One insect used for one IAP.

${ }^{\mathrm{d}}$ Four insects used for 3-day IAP. effect" attained by the removal of the inoculated leaf for qPCR analyses after 3 and 7 days of IP. These time periods are probably not long enough for the bacterium to leave the leaf petiole and reach the stem.

In this work, we confirmed the suitability of the excised leaf assay to study the initial steps of ' $\mathrm{Ca}$. L. asiaticus' infection in citrus. On young and soft leaves, the efficiencies of successful inoculation were higher than on mature and hardened leaves. The relatively short longevity found initially was overcome by the maintenance of a branch segment at the base of the leaf petiole. With this procedure, higher infection frequencies, in general, were obtained with the use of four adult psyllids allowed to feed on leaves for 3 to 7 days. It is possible, however, that transmission efficiency may be different between vector or pathogen populations in different geographical areas, or with different experimental conditions. Excised tissue even younger than that tested in this work (namely, newly emerged about 5-cm-long soft, immature shoots) was also tested. Although useful in studies involving ' $\mathrm{Ca}$. L. asiaticus' acquisition (Lopes et al. 2013), a phenomenon that occur in minutes or hours (Xu et al. 1988), the even shorter survival period (no longer than 3 to 4 days) limited their use for ' $C a$. L. asiaticus' inoculation (results not shown).

\section{Acknowledgments}

We thank L. W. Timmer, Professor Emeritus, University of Florida, Lake Alfred; J. R. S. Lopes, ESALQ, University of Sao Paulo; and L. Peña, Fundecitrus, for useful suggestions during manuscript preparation.

\section{Literature Cited}

Ammar, E. D., Hall, D. G., and Alvarez, J. M. 2015. Effect of cyantraniliprole, a novel insecticide, on the inoculation of Candidatus Liberibacter asiaticus associated with citrus huanglongbing by the Asian citrus psyllid (Hemiptera: Liviidae). J. Econ. Entomol. 108:399-404.

Ammar, E.-D., Walter, A. J., and Hall, D. G. 2013. New excised-leaf assay method to test inoculativity of Asian citrus psyllid (Hemiptera: Psyllidae) with Candidatus Liberibacter asiaticus associated with citrus huanglongbing disease. J. Econ. Entomol. 106:25-35.

Bassanezi, R. B., Montesino, L. H., Gasparoto, M. C. G., Bergamin Filho, A., and Amorim, L. 2011. Yield loss caused by huanglongbing in different sweet orange cultivars in São Paulo, Brazil. Eur. J. Plant Pathol. 130:577-586.

Capoor, S., Rao, D., and Viswanath, S. 1967. Diaphorina citri Kuway., a vector of the greening disease of citrus in India. Indian J. Agric. Sci. 37:572-579.

Coletta-Filho, H. D., Targon, M. L. P. N., Takita, M. A., De Negri, J. D., Pompeu, J., Machado, M. A., Amaral, A. M., and Muller, G. W. 2004. First report of the causal agent of huanglongbing ("Candidatus Liberibacter asiaticus") in Brazil. Plant Dis. 88:1382.

Fundecitrus. 2016. Alta taxa de eliminação de plantas doentes mantém incidência de HLB estável. Online publication. http://www.fundecitrus.com.br/comunicacao/ noticias/integra/alta-taxa-de-eliminacao-de-plantas-doentes-mantem-incidenciade-hlb-estavel/408

Gottwald, T. R. 2010. Current epidemiological understanding of citrus huanglongbing. Annu. Rev. Phytopathol. 48:119-139.

Halbert, S. E., and Manjunath, K. L. 2004. Asian citrus psyllids (Sternorrhyncha: Psyllidae) and greening disease of citrus: A literature review and assessment of risk in Florida. Fla. Entomol. 87:330-353.

Hoffman, M. T., Doud, M. S., Williams, L., Zhang, M.-Q., Ding, F., Stover, E., Hall, D., Zhang, S., Jones, L., Gooch, M., Fleites, L., Dixon, W., Gabriel, D., Gabriel, D., and Duan, Y. 2013. Heat treatment eliminates "Candidatus Liberibacter asiaticus" from infected citrus trees under controlled conditions. Phytopathology 103:15-22.

Li, W., Hartung, J. S., and Levy, L. 2006. Quantitative real-time PCR for detection and identification of Candidatus Liberibacter species associated with citrus huanglongbing. J. Microbiol. Methods 66:104-115.

Lopes, S. A., Bertolini, E., Frare, G. F., Martins, E. C., Wulff, N. A., Teixeira, D. C., and Fernandes, N. G. 2009. Graft transmission efficiencies and multiplication of "Candidatus Liberibacter americanus" and " $\mathrm{Ca}$. Liberibacter asiaticus" in citrus plants. Phytopathology 99:301-306.

Lopes, S. A., Frare, G. F., Yamamoto, P. T., Ayres, A. J., and Barbosa, J. C. 2007. Ineffectiveness of pruning to control citrus huanglongbing caused by Candidatus Liberibacter americanus. Eur. J. Plant Pathol. 119: 463-468.

Lopes, S. A., Luiz, F. Q. B. F., Martins, E. C., Fassini, C. G., Sousa, M. C., Barbosa, J. C., and Beattie, G. A. C. 2013. "Candidatus Liberibacter asiaticus" titers in citrus and acquisition rates by Diaphorina citri are decreased by higher temperature. Plant Dis. 97:1563-1570.

Teixeira, D., Danet, J. L., Eveillard, S., Martins, E. C., Jesus Junior, W. C., Yamamoto, P. T., Lopes, S. A., Bassanezi, R. B., Ayres, A. J., Saillard, C., and Bové, J. M. 2005a. Citrus huanglongbing in São Paulo State, Brazil: 
PCR detection of the 'Candidatus' Liberibacter species associated with the disease. Mol. Cell. Probes 19:173-179.

Teixeira, D., Saillard, C., Eveillard, S., Danet, J. L., da Costa, P. I., Ayres, A. J., and Bové, J. 2005b. "Candidatus Liberibacter americanus", associated with citrus huanglongbing (greening disease) in Sao Paulo State, Brazil. Int. J. Syst. Evol. Microbiol. 55:1857-1862.

Tsai, J. H., and Liu, Y. H. 2000. Biology of Diaphorina citri (Homoptera: Psyllidae) on four host plants. J. Econ. Entomol. 93:1721-1725.

Xu, C., Xia, Y., Li, K., and Ke, C. 1988. Further study of the transmission of citrus huanglongbing by a psyllid, Diaphorina citri Kuwayama. Pages 243-248 in: Proc. 10th Conf. Int. Organ. Citrus Virol. Valencia, Spain. L. Navarro, L. W. Timmer, and S. M. Garnsey, eds.
Yamamoto, P. T., Felippe, M. R., Garbim, L. F., Coelho, J. H. C., Ximenes, N. L., and Martins, E. C. 2006. Diaphorina citri (Kuwayama) (Hemiptera: Psyllidae): Vector of the bacterium Candidatus Liberibacter americanus. Page 96 in: Proc. Huanglongbing Green. Int. Workshop, Ribeirão Preto, SP, Brazil

Yang, C., Powell, C. A., Duan, Y., Shatters, R., Fang, J., and Zhang, M. 2016. Deciphering the bacterial microbiome in huanglongbing-affected citrus treated with thermotherapy and sulfonamide antibiotics. PLoS One 11: $\mathrm{e} 0155472$.

Zhang, M., Guo, Y., Powell, C. A., Doud, M. S., Yang, C., and Duan, Y. 2014 Effective antibiotics against "Candidatus Liberibacter asiaticus" in HLBaffected citrus plants identified via the graft-based evaluation. PLoS One 9: $17-21$. 